\title{
Bioartificial organs and acceptable risk
}

\author{
David Hunkeler, Anthony M. Sun, Gregory S. Korbutt, Ray V. Rajotte, Ronald G. Gill, Riccardo Calafiore, and Philippe Morel
}

The risks incurred with whole-organ xenotransplantation differ substantially from those associated with cellular transplants, particularly if the latter are contained within an immunoisolation barrier provided by encapsulation. Given that the forecasted demand for bioartificial organs-cellular, autograft, allograft, and xenograft transplants - is estimated to be in the billions of dollars per annum ${ }^{1}$, with applications including a range of hormone-deficiency and neurological diseases ${ }^{2}$, one must question what is an appropriate level of societal risk, and what developmental path should be advocated to responsibly implement this technology. Ameliorating the blood glucose control in type 1 diabetes through islet transplantation, to give just one example, would help control the many secondary effects of the disease, including retinopathy, kidney failure, microangiopathy, and arteriosclerosis, with a concomitant reduction in health-care costs.

In 1998, Fritz Bach and Harvey Fineberg called for a comprehensive moratorium on xenotransplantation ${ }^{3}$. We believe the moratorium is relevant to whole-organ transplantation but would urge that cellular transplantation be handled separately, because the risks presented by the two technologies are different. Cellular immunoisolated xenografts should entail less risk than do wholeorgan transplants. Since the actual biological risk, i.e., the exact pathogen, is unknown, we cannot "know" that the immunoisolated graft contains less risk. But immunoisolation provides a theoretical benefit unless further data are generated.

D. Hunkeler is professor, laboratory of polymers and biomaterials, Swiss Federal Institute of Technology, Lausanne, Switzerland (David.Hunkeler@epfl.ch); A. Sun is professor, department of physiology, University of Toronto, Toronto, ON, Canada; G. Korbutt is assistant professor of surgery, surgical-medical research institute, University of Alberta, Edmonton, $A B$, Canada; R. Rajotte is professor of surgery and medicine, surgical-medical research institute, University of Alberta, Edmonton, AB, Canada; R. Gill is professor, Barbara Davis Center for Childhood Diabetes, Denver, CO; R. Calafiore is director, laboratory for the study and transplant of pancreatic islets, University of Perugia, Perugia, Italy; Ph. Morel is director, clinic and polyclinic for digestive surgery, transplantation unit, University Hospital of Geneva, Geneva, Switzerland.
Bioartificial organs are (1) viral protective, that is, the membrane controls ingress/egress; (2) antigen blocking, in that surface modifications change the host-contacting surface, blocking the antigen attachment-endothelial cell activationhyperacute

\section{The moratorium on whole- organ xenotransplantation should not be extended to xenograft-based bioartificial organs.}

rejection cascade; (3) retrievable, meaning that immunoisolation devices/bioartificial organs can be designed for retrievability; (4) isolating, in that macro/micro device cascades can be constructed to provide cell isolation; (5) controllable, that is, cells can be genetically modified with suicide genes; and (6) robust, that is, the transplantation site can be varied for bioartificial organs (e.g., use an immunoprivileged site).

\section{Bioartificial clinical trials}

Xenograft therapy has been applied extensively in clinical settings. Thousands of patients have received successful porcine skin transplants, type 1 diabetic patients have been implanted with porcine islets ${ }^{4}$, and patients with chronic glomerulonephritis have been connected for short periods of time to porcine kidneys ${ }^{5,6}$. Therefore, the pig should not be rejected outright as a donor, even though some porcine endogenous retroviruses (PERVs), found in their chromosomes, cannot be eliminated ${ }^{7}$. Recent encouraging studies have addressed this issue ${ }^{14}$. Patience et al. ${ }^{8}$ analyzed DNA isolated from patients' peripheral blood mononuclear cells and found no evidence of PERV DNA in two renal dialysis patients whose circulations were linked extracorporeally to porcine kidneys. In addition, no seroconversion for PERV-specific antibodies was observed ${ }^{8}$. Similarly, Heneine et al. ${ }^{9}$ were unable to detect markers for PERV infection in 10 patients who were transplanted with fetal porcine islets despite the evidence of extended exposure to porcine cells (10 years) and concomitant immunosuppressive therapy. Additionally, risk assessment can be performed before transplantation for cellular grafts such as islets. Indeed, preliminary evaluations have already been carried out; extracorporeal xenograft-based bioartificial livers (BAL) are in phase III clinical trials ${ }^{10,15}$.
Clinical testing has also been carried out on double immunoisolated bioartificial pancreases consisting of microencapsulated islets housed in a vascularized chamber ${ }^{11}$. Though applied at subtherapeutic doses, the islets were shown to survive and normoglycemia was established for a limited period in one of two patients. This work gave impetus to long-term xenograft trials (pig-tomonkey) that resulted in diabetes reversal for up to two years ${ }^{12}$. The use of a hollowfiber device to isolate genetically modified cells for pain control in terminally ill patients ${ }^{13}$ has also been evaluated in the clinic. As a safeguard in the event of transplant failure, the termination of cell secretion can be made possible by incorporating a suicide gene in the donor tissue. In addition, cell transplants can be designed for rapid retrievability without major surgery. While these safety measures do not eliminate all the risks involved in cellular xenotransplantation, they do demonstrate that exposure to risk, even if identified posttransplantation, can be managed responsibly.

\section{Conclusions}

We believe that research on xenotransplantation in concordant and discordant animal models should proceed. Cellular xenograft transplantation should follow the clinical demonstration of concept and evaluation of bioartificial organ function in auto- and allografts. Cellular and whole-organ xenotransplants, however, involve unique recipient and societal risks and should, therefore, be regulated independently. The risks of cellular xenotransplantation can be assessed without threatening the public health, and thus the moratorium on whole-organ xenotransplantation should not be extended to xenograft-based bioartificial organs.

1. Langer, R. \& Vacanti, J.P. Science 260, 920 (1993)

2. Lanza, R.P. et al. Nat. Biotechnol., 14, 1107 (1996)

3. Bach, F.H. \& Fineberg, H.V. Nature 391, 326 (1998).

4. Groth, C.G. et al. Lancet 344, 1402 (1994).

5. Breimer, M.E. et al. Xenotransplantation 3, 328 (1996).

6. Rydberg, L. et al. Xenotransplantation 3, 340 (1996).

7. Masood, E. Nature 394, 513 (1998).

8. Patience, C. et al. Lancet 352, 699 (1998).

9. Heneine, W.H. et al. Lancet 352, 695 (1998)

10. Demetriou, A.A. Ann. NY Acad. Sci. 875, 350 (1999).

11. Calafiore, R. et al. Ann. NY Acad. Sci. 830, 313 (1998).

12. Sun, Y.L. et al. J. Clin. Invest. 98, 1417 (1996)

13. Aebischer, P. \& Borkenhager, M. MRS Bulletin 21, 59 (1996).

14. Bradis, K. et al.. Science 285, 1236 (1999).

15. Lohr, M. et al. J. Mol. Med. 77, 393 (1999). 